\title{
Are two spaces better than one? The effect of spacing following periods and commas during reading
}

\author{
Rebecca L. Johnson ${ }^{1} \cdot$ Becky Bui $^{1} \cdot$ Lindsay L. Schmitt ${ }^{1}$
}

Published online: 24 April 2018

(C) The Psychonomic Society, Inc. 2018

\begin{abstract}
The most recent edition of the American Psychological Association (APA) Manual states that two spaces should follow the punctuation at the end of a sentence. This is in contrast to the one-space requirement from previous editions. However, to date, there has been no empirical support for either convention. In the current study, participants performed (1) a typing task to assess spacing usage and (2) an eye-tracking experiment to assess the effect that punctuation spacing has on reading performance. Although comprehension was not affected by punctuation spacing, the eye movement record suggested that initial processing of the text was facilitated when periods were followed by two spaces, supporting the change made to the APA Manual. Individuals' typing usage also influenced these effects such that those who use two spaces following a period showed the greatest overall facilitation from reading with two spaces.
\end{abstract}

Keywords eye-tracking $\cdot$ reading $\cdot$ punctuation $\cdot$ spacing

There has been a long debate on the topic of how many spaces should follow a period at the end of a sentence. Professionals and amateurs in a variety of fields have passionately argued for either one or two spaces following this punctuation mark (Gonzalez, 2014; Manjoo, 2011; McArdle, 2011). Some arguments for using one space at the end of a sentence have centered on aesthetics (Manjoo, 2011), while others defend the one-space rule simply because it saves time and effort (Walker, 2011). On the other hand, advocates for the twospace rule have insisted that the extra space enhances the ease of text readability and that it is considerate to include the extra space for the reader (Lee, 2011; Wiederkehr, 2009). This debate has been revisited in response to the recent changes made to the Sixth Edition of the American Psychological Association (APA) Manual (2010), which now states that two spaces should follow a sentence-completing punctuation mark when creating a manuscript because "spacing twice after punctuation marks at the end of a sentence aids readers of draft manuscripts" (p. 88). This formatting detail has been altered from the Fifth Edition of the manual (2001), which required only one space. According to APA's website and blog devoted

Rebecca L. Johnson

rjohnso1@skidmore.edu

1 Department of Psychology, Skidmore College, Saratoga Springs, NY 12866, USA to changes in the manual, they justify this change by stating that two spaces should now be used "for ease of reading comprehension" and "to ease...reading by breaking up the text into manageable, more easily recognizable chunks" (Wiederkehr, 2009). However, to date, there has been no direct empirical evidence in support of these claims, nor in favor of the one-space convention.

Theoretical arguments can be made on both sides. Past work demonstrates that readers receive parafoveal information from the word to the right of fixation before directly fixating on it (see Schotter, Angele, \& Rayner, 2012, for a review). This parafoveal preview benefit is even present when fixating on the last word in a sentence, where the extraction of parafoveal information occurs across sentence boundaries (Payne \& Stine-Morrow, 2012; White, Warren, \& Reichle, 2011). Since the amount of facilitation that we receive from the right of fixation is a function of how far into the parafovea the information is (Legge, Mansfield, \& Chung, 2001; Rayner, McConkie, \& Zola, 1980), using only one space after a punctuation mark may enable the reader to capture more characters within their perceptual span and begin processing them sooner. However, it could be argued that using two spaces after a punctuation mark may decrease the lateral interference (i.e., difficulty in target identification as a function of surrounding items) that the reader experiences from adjacent letters, thereby facilitating processing (e.g., Grainger, Tydgat, \& Issele, 2010; Levi, 2008; Pelli, Tillman, Freeman, 
Su, Berger, \& Majaj, 2007; van den Boer \& Hakvoort, 2015; Wolford \& Chambers, 1983). Lateral interference is a function of spacing; the more space that appears between target and filler items, the easier it is to identify the target (Wolford \& Chambers, 1983; Yu, Cheung, Legge, \& Chung, 2007).

Although previous research has been conducted on how reading is affected by inter-word spacing (Cui, Drieghe, Bai, Yan, \& Liversedge, 2014; Drieghe, Brysbaert, \& Desmet, 2005; Jacobs, 1987; Johnson \& Eisler, 2012; Malt \& Seamon, 1978; McGowan, White, \& Paterson, 2015; Morris, Rayner, \& Pollatsek, 1990; Paterson \& Jordan, 2010; Perea \& Acha, 2009; Pollatsek \& Rayner, 1982; Rayner, Fischer, \& Pollatsek, 1998), inter-letter spacing (Paterson \& Jordan, 2010; Perea, Moret-Tatay, \& Gomez, 2011; Van Overshelde \& Healy, 2005; Yu et al., 2007), interline spacing (Van Overshlelde \& Healy, 2005), and having punctuation vs. not (Hill \& Murray, 2000; Hirotani, Frazier, \& Rayner, 2006; Pynte \& Kennedy, 2007), none has investigated the effects of spacing following punctuation marks on reading performance. The aim of the present study was to do just that.

Although the current study is the first to directly explore spacing following punctuation (specifically for periods and commas), several previous studies do provide some insight on the importance of spacing in text. Ultimately, research on the effects of inter-word spacing and word boundary information has demonstrated that space information is extracted early in the reading process (Pollatsek \& Rayner, 1982) and plays a major role in helping readers identify words and direct eye movements (Perea \& Acha, 2009; Rayner et al., 1998). These conclusions are based on the finding that readers experience difficulty reading text where inter-word spaces have been filled in with another symbol (Epelboim, Booth, Ashkenazy, Taleghani, \& Steinman, 1997; Johnson \& Eisler, 2012; Malt \& Seamon, 1978; McConkie \& Rayner, 1975; McGowan, White, Jordan, \& Paterson, 2014; Morris et al., 1990; Pollatsek \& Rayner, 1982; Rayner et al., 1998; Spragins, Lefton, \& Fischer, 1976) or word boundaries have been removed altogether (Drieghe, Fitzsimmons, \& Liversedge, 2017; Johnson \& Eisler, 2012; Paterson \& Jordan, 2010; Perea \& Acha, 2009; Rayner et al., 1998; Spragins et al., 1976). Studies that have explored the effects of increasing the inter-word spacing have shown mixed effects. For example, Drieghe et al. (2005) found that inserting two spaces between words facilitated reading when compared to inserting a single space, which they attribute to reduced lateral interference. Rayner et al. (1998, Experiment 2), however, showed negligible differences in reading rates and fixation durations when increasing the inter-word spacing, and McGowan et al. (2015) report a cost in overall reading times when using wide or expanded spacing.

In the current study, participants read paragraphs that included either one or two spaces following periods and commas. Varying the number of spaces following periods allowed us to directly test how reading performance might differ as a function of the currently debated spacing conventions. We manipulated the spacing following commas to explore whether increased spacing facilitated reading across punctuation types. Reading performance was assessed with comprehension questions and overall reading speed, as well as by analyzing eye-movements, a methodology that has been used for decades to explore cognitive processing within normal silent reading (see Rayner, 1998, 2009, for reviews). In light of the fact that people differ (sometimes passionately) with regards to their spacing preference, we also performed a typing task to explore reading effects as a function of spacing usage.

\section{Method}

\section{Participants}

Participants in the present study included 60 students at Skidmore College who were native speakers of American English and had normal or corrected-to-normal vision. Participants received one hour of research credit for their participation.

\section{Materials and apparatus}

The present study included two components: (1) a typing task to measure participants' usage of spacing following commas and periods and (2) a paragraph reading study utilizing eyetracking methodology.

\section{Typing task}

The typing task consisted of a short sample paragraph (97 words in length) which participants were asked to type on a Dell PC computer with a 19-inch monitor as it was orally dictated to them. Participants were told that accuracy, not speed, was being measured. A correctly typed paragraph would include 5 periods (of which all but the last could be used to determine spacing usage) and 9 commas. Of the 60 participants, 39 were categorized as "One-Spacers" (they consistently used one space after periods), and 21 were categorized as "Two-Spacers" (they consistently used two spaces after periods). All participants used one space following commas.

\section{Eye-tracking study}

Following the typing task, participants were asked to silently read 20 paragraphs (in addition to 1 practice paragraph) presented on a 21 inch NEC Accusync 120 monitor interfaced 
with a Dell computer with an Intel Core2 Duo processor. While they were reading, their eye-movements were recorded with an Eyelink 1000 which recorded the position of their eye every millisecond. Chin and forehead rests were used to minimize the reader's head movements. Although reading took place binocularly, eye movements were only recorded from the participant's right eye.

The number of spaces following periods ( 1 vs. 2 ) and the number of spaces following commas (1 vs. 2) were both manipulated as within-subjects and within-items variables. Each experimental paragraph was presented in one of four counterbalanced conditions: (1) one space after periods and commas; (2) one space after periods, two spaces after commas; (3) two spaces after periods, one space after commas; and (4) two spaces after periods and commas. These manipulations were also counterbalanced across participants, with each participant reading 5 paragraphs in each of the 4 conditions.

The 20 experimental paragraphs were 71 to 166 words in length $(M=116)$ and were presented in 14 point Courier New font. Inter-line spacing was quadruple spaced to ensure that eye fixations could be accurately assigned to each line of text when manual adjustments were necessary during data analysis. In order to adequately explore the influence of spacing following periods and commas, each of the paragraphs contained multiple periods $(M=8.05$; range $=5$ to 11$)$ and commas $(M=5.10$; range $=3$ to 8$)$. With the exception of the paragraph-final period, these were all used as regions of interest in the local analyses. In all, there were a total of 141 periods and 102 commas read by each participant.

\section{Procedure}

Upon arriving to the experiment, each participant read and signed the informed consent document and was given instructions for the study. The participant first completed the typing task, which lasted approximately 10 minutes. The participant then took part in the eye-tracking study where he or she was instructed to rest his or her head on the chin and forehead rests. The eyetracker was calibrated to the participant's eye by having him or her focus on a circle that moved to 13 points around the computer screen. Following calibration, a black box was presented in the upper left-hand corner of the computer screen corresponding to the location of the first letter of the first word in the paragraph. Once a stable fixation was achieved on the black box, a paragraph appeared on the screen. Before reading the experimental trials, participants first read a practice paragraph to ensure their level of comfort with the procedure. The experimental session consisted of 20 paragraphs presented in random order. Each participant sat $83 \mathrm{~cm}$ from the monitor. At this distance, 4 characters equaled 1 degree of visual angle.

During the eye-tracking experiment, each participant was instructed to silently read the paragraphs presented on the computer screen. Each paragraph was presented in one of the four experimental conditions such that the number of spaces following periods and commas varied. Participants read each paragraph and pressed a button to indicate when they had finished reading, at which point the paragraph disappeared. Each paragraph was followed by a question to ensure that participants were reading for comprehension. Participants were instructed to indicate which of the two possible answers presented on the screen was correct by pressing the corresponding button on a response box. The accuracy of the initial calibration was then checked, and the process repeated until all experimental items were completed. The eye-tracking experiment took approximately 30 minutes. Each participant was then given a written debriefing form and compensated with one hour of research credit.

\section{Results}

\section{Global measures}

To examine differential processing across the entire paragraph as a function of the four reading conditions, two global measures were analyzed: overall reading speed (as measured by the average number of words per minute (WPM)) and comprehension accuracy. WPM measurements that were more than two standard deviations above the participant's mean were removed from data analyses. This resulted in the elimination of $3.7 \%$ of the data in this measure. Table 1 presents the means for these global measures as a function of spacing condition and spacing usage.

Table 1 Mean Reading Speed and Comprehension Accuracy as a Function of Spacing Condition and Spacing Usage

\begin{tabular}{|c|c|c|c|c|c|c|c|c|}
\hline & \multicolumn{4}{|c|}{ "One-Spacers" } & \multicolumn{4}{|c|}{ “Two-Spacers" } \\
\hline & $1 \mathrm{P} 1 \mathrm{C}$ & $1 \mathrm{P} 2 \mathrm{C}$ & $2 \mathrm{P} 1 \mathrm{C}$ & $2 \mathrm{P} 2 \mathrm{C}$ & $1 \mathrm{P} 1 \mathrm{C}$ & $1 \mathrm{P} 2 \mathrm{C}$ & $2 \mathrm{P} 1 \mathrm{C}$ & $2 \mathrm{P} 2 \mathrm{C}$ \\
\hline Reading Speed (WPM) & $291(85)$ & $286(82)$ & $288(78)$ & $292(79)$ & $305(72)$ & $304(70)$ & $314(71)$ & $301(74)$ \\
\hline Comprehension Accuracy (\%) & $88.7(31.8)$ & $89.2(31.1)$ & $89.2(31.1)$ & $86.7(34.1)$ & $93.3(25.2)$ & $89.2(31.2)$ & $89.4(30.9)$ & $88.6(32.0)$ \\
\hline
\end{tabular}

Note. Paragraphs were presented in one of four conditions where the spacing following periods ( $1 \mathrm{vs} .2,1 \mathrm{P}$ vs. $2 \mathrm{P}$ respectively) and the spacing following commas (1 vs. $2,1 \mathrm{C}$ vs. $2 \mathrm{C}$ respectively) was manipulated. Standard deviations are reported in parentheses 
Global measures were analyzed using a mixed effects regression model from the lmerTest package (Kuznetsova, Brockhoff, \& Christensen, 2015) within the R environment for Statistical Computing (R Development Core Team, v. 3.2.0, 2015. A full random structure was initially specified for subjects and items, but when this model did not converge, the final model used was one that included random intercepts (but not slopes) for subjects and items. Period spacing type (1 vs. 2) and comma spacing type (1 vs. 2) were effect coded (where paragraphs with 1 space following punctuation marks were coded as -.5 and paragraphs with 2 spaces following punctuation marks were coded as .5) and entered as fixed effects. In addition, the participant's period spacing usage as measured by the typing task was also effect coded (where "one-spacers" were coded as -.5 and "two-spacers" were coded as .5) and included in the model as a fixed effect. The interactions between these fixed effects were also included in the model.

In the analysis of reading speed, although there was not an overall significant effect of period spacing $(\beta=3.10, S E=$ $2.20, t=1.41, p=.160)$ or typing condition $(\beta=17.62, S E$ $=18.85, t=0.94, p=.354)$, there was a significant effect of comma spacing $(\beta=-4.99, S E=2.19, t=-2.27, p=.023)$ such that readers read paragraphs faster when they were written with only one space after the commas, as is the common convention. Notably, there was also a significant three-way interaction among the fixed effects $(\beta=-23.24, S E=8.88, t$ $=-2.62, p=.009)$. Although the type of spacing following punctuation marks did not seem to have an effect on those individuals who type with one space after a period, those who type with two spaces after a period had greater reading speed when paragraphs were presented in the same way in which they type: with two spaces following periods and one space following commas ${ }^{1}$. See Figure 1 for a graphical representation of this interaction.

Comprehension accuracy was high across all participants $(M=89 \%$; range $=79 \%$ to $100 \%)$ and did not differ as a function of period spacing $(\beta=-.237, S E=.237, z=-.997$, $p=.319)$, comma spacing $(\beta=-.298, S E=.237, z=-1.26, p=$ $.209)$, or the participant's period spacing usage $(\beta=.241, S E=$ $.236, z=1.02, p=.309)$. Furthermore, none of the interactions were statistically significant (all $p s>.362$ ).

\footnotetext{
${ }^{1}$ Reading speed (in WPM) was normally distributed and so the raw untransformed data was used in the LMM analysis. However, even when the reading times were log transformed (as was the practice in analyzing the local effects where fixation durations were positively skewed), the pattern of significant findings was identical. Specifically, there was not an overall significant effect of period spacing $(\beta=.012, S E=.008, t=1.52, p=.129)$ or typing condition $(\beta=.070, S E=.062, t=1.12, p=.270)$, but there was a significant effect of comma spacing $(\beta=-0.17, S E=.008, t=-2.27, p=.023)$ and a significant three-way interaction among the fixed effects $(\beta=-.081, S E=.031, t=-2.67, p$ $=.008)$
}

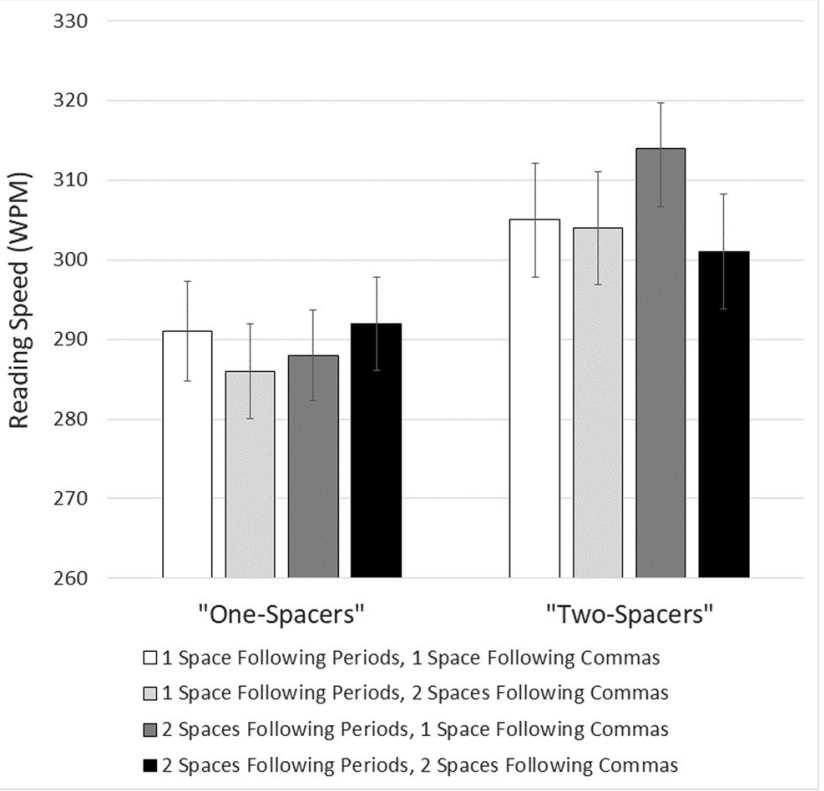

Fig. 1 Paragraph reading speed as a function of spacing condition and spacing usage. Error bars represent the standard error of the mean for each condition

\section{Local measures}

One benefit to recording eye-movements is that we are able to capture online processing, thereby evaluating the time course of processes as they unfold (Rayner, 1998, 2009). In order to more fully examine the effect of spacing following punctuation on reading, we analyzed local measures of processing on the two-word region surrounding each of the manipulated punctuation marks. That is, the target regions included the word immediately preceding the punctuation, the punctuation mark itself, and the word immediately following the punctuation.

For the local measures, regions where the participant blinked or the eye-tracker lost track of the participant's eye on the two words preceding or following the period/comma were removed from data analyses. In cases where there were two fixations on adjacent letters and one of the fixations was extremely short (less than $80 \mathrm{~ms}$ ), the two fixations were pooled. Extremely short isolated fixations (those less than 80 $\mathrm{ms}$ ) and extremely long fixations (those greater than $1000 \mathrm{~ms}$ ) were eliminated from the data prior to analyses. These trimming procedures resulted in the elimination of $8.68 \%$ of the data.

Dependent measures that were analyzed included early measures of processing (specifically, the probability of skipping the target region, the first fixation duration on the region, the first pass reading time on the region before leaving it, and the number of fixations made on the region during the first pass reading of it), and later measures of processing (specifically, the percentage of regressions made back into the region 
after leaving it and the total time spent on the target region including any regressions made back to it).

\section{Effects near the period}

Effects seen in the two-word regions near the periods were analyzed using linear mixed effects regression models from the lmerTest package (Kuznetsova et al., 2015) within the R environment for Statistical Computing (R Development Core Team, v. 3.2.0, 2015). Again, a full random structure was initially specified for subjects and items, but when this model did not converge, the final model used was one that included random intercepts (but not slopes) for subjects and items. Period spacing type (1 vs. 2) was effect coded (where paragraphs with 1 space following periods were coded as -.5 and paragraphs with 2 spaces following periods were coded as .5) and entered as a fixed effect. The participant's period spacing usage as measured by the typing task was also effect coded (where "one-spacers" were coded as -.5 and "two-spacers" were coded as .5) and included in the models as a fixed effect, along with its interaction with period spacing. Fixation duration measures were positively skewed and were thus log transformed prior to analyses. The two binary dependent measures (e.g., skipping rate, regressions in) were analyzed using mixed-effects logistic regression, with the same random and fixed effects.

The effects of period spacing from these mixed effects analyses are presented in Table 2 . Across all of the early measures, there was a significant effect of period spacing. Paragraphs written with two spaces following each period led to greater skipping rates, shorter first fixation durations, shorter first pass times, and fewer first pass fixations compared to paragraphs written with only one space following each period. There were also significant effects seen in later measures; paragraphs written with two spaces following each period led to shorter total fixation durations compared to paragraphs written with one space following each period. There was, however, no significant effect seen in the percentage of regressions made back to the target region. There was not a significant difference in eye movement measures between "one-spacers" and "two-spacers" (all $p \mathrm{~s}>.091$ ), nor did typing preference interact with the period spacing effect (all $p \mathrm{~s}>$ $.315)$.

\section{Effects near the comma}

Effects seen in the two-word regions near the commas were also analyzed using mixed effects regression models using the same structure of fixed and random effects used when analyzing the two-word regions near the periods. Fixation duration measures were again positively skewed and were thus log transformed prior to analyses. The effects of comma spacing from these mixed effects analyses are presented in Table 3. In the early measures, there was not a significant effect of comma spacing on the fixation duration measures nor on the number of first pass fixations that readers made, although there was a trend for readers to spend less time on target regions when there were two spaces following the commas. The effect of comma spacing was also not significant across any of the later measures.

First pass times in the comma region were significantly longer $(\beta=-0.099, S E=.043, t=-2.32, p=.024)$ for those who were identified as "one-spacers" during the typing task $(M=311, S D=188)$ than for "two-spacers" $(M=276, S D=$ 166). Similarly, more first pass fixations were made in the comma region $(\beta=-0.124, S E=0.061, t=-2.05, p=.045)$ by "one-spacers" $(M=1.07, S D=.81)$ than by "two-spacers" $(M=.94, S D=.76)$. There were no other differences between these two typing groups in any other measure (all $p \mathrm{~s}>.102$ ). Finally, there was a two-way interaction in the skipping measure $(\beta=-0.341, S E=0.132, z=-2.59, p=.010)$ such that while "one-spacers" were more likely to skip the target region if it had two spaces after the comma $(M=24.6 \%, S D=43.1 \%)$ than if it only had one space after it $(M=20.3 \%, S D=40.3 \%)$, "two-spacers" were less likely to skip the target region if it had two spaces after the comma $(M=26.4 \%, S D=44.1 \%)$ than if it only had one space after it $(M=27.6 \%, S D=44.7 \%)$. None of the other two-way interactions were significant (all $p \mathrm{~s}>$ .103).

\section{Discussion}

The eye movement record indicated that reading performance is affected by the number of spaces following periods. Specifically, when text was presented with two spaces following the periods, readers were more likely to skip the punctuation region, make fewer fixations on it, and fixate for a shorter duration than when the periods were only followed with one space. These effects were not dependent on one's typing preference; even those who type with only one space following periods showed early facilitation in processing the region near the period when it was followed by two spaces. However, period spacing was an early but short-lived effect. It did not affect the likelihood of regressing back to the period region, nor did it have strong effects in the global measures. Comprehension accuracy was high across all conditions and did not differ as a function of the number of spaces following the punctuation marks, and reading speed only increased following two spaces for those individuals who also type that way. However, the passages used in the current study were relatively short and may not have been long enough or difficult enough to detect subtle global differences due to punctuation spacing. Future research could explore this in more depth. 
Table 2 Means (and Standard Deviations) on the Period Region as a Function of Period Spacing

\begin{tabular}{lccrrrr}
\hline & 1 Space After Periods & 2 Spaces After Periods & $\beta$ & $S E$ & $t$ or $z$ statistic & $p$-value \\
\hline Early Measures & & & & & \\
$\quad$ Skipping Rate (\%) & $22.4(41.7)$ & $27.9(44.9)$ & .325 & .055 & 5.97 \\
$\quad$ First Fixation (ms) & $224(83)$ & $219(85)$ & -.027 & .009 & -3.21 & -001 \\
First Pass (ms) & $290(173)$ & $283(171)$ & -.037 & .012 & -2.99 \\
$\quad$ Number of First Pass Fixations & $1.00(0.73)$ & $0.92(0.74)$ & -.085 & .016 & -5.28 \\
Late Measures & & & -001 & .003 \\
$\quad$ Regressions In (\%) & $15.6(36.3)$ & $15.5(36.2)$ & -.017 & .075 & -0.22 \\
$\quad$ Total Time (ms) & $372(249)$ & $362(237)$ & -.037 & .014 & -2.69 \\
\hline
\end{tabular}

Although this facilitative spacing effect occurred for periods, it did not transfer to commas. In fact, overall reading speeds were slowed when paragraphs were presented with two spaces following commas and those who type with twospaces following a period were more likely to fixate these regions that were presented against convention. Thus, we cannot assume that increased punctuation spacing will always lead to facilitation in processing the text.

Further research should investigate why reading is facilitated when periods are followed by two spaces. For example, consider the following non-mutually exclusive possibilities. First, as mentioned earlier, the inclusion of two spaces following punctuation marks may reduce the amount of lateral interference that foveal or parafoveal letters experience (Cui et al., 2014; Drieghe et al., 2005). Second, reading text with two spaces following punctuation could also aid in identifying and isolating processing units (Jacobs, 1987), in this case, the end of a sentence. Third, the additional space may in some way facilitate wrap-up effects which are typically found near commas and periods (Hirotani et al., 2006; Rayner, Kambe, \& Duffy, 2000; Warren, White, \& Reichle, 2009).

It should be noted that the paragraphs used in the current experiment were presented in a monospaced fixed font (i.e., where each character is of equal width). Many other fonts used by word processers today utilize proportional fonts (i.e., the computer adjusts for the unique width of each character). Slattery, Yates, and Angele (2016) recently found that interword spacing effects differed depending on whether a fixed width font on a proportional width font was used. Thus, it is possible that the effects of punctuation spacing seen in the current experiment may differ when presented in other font conditions (or other writing systems). However, if the facilitation from two spaces is due in whole or in part to increasing the space relative to other spaces (e.g., to indicate not only the end of a word, but also the end of a sentence), then two spaces should facilitate reading even when text is presented in a proportional font where a single space is the same size regardless of whether it follows a punctuation mark or not.

Finally, it is worth considering that effects of punctuation spacing may be susceptible to individual differences. In fact, in the current study, there were differences noted between those that type using a one-space convention and those that type using a two-space convention. Future research might explore other individual differences. For example, those with visual deficits, such as older readers, may especially benefit from increased spacing near periods as they have previously been shown to have more pronounced effects of spacing in other reading contexts (McGowan et al., 2014; Rayner, Yang, Schuett, \& Slattery, 2013; though see McGowan et al., 2015). Furthermore, Zorzi et al. (2012) noted advantageous effects of

Table 3 Means (and Standard Deviations) on the Comma Region as a Function of Comma Spacing

\begin{tabular}{|c|c|c|c|c|c|c|}
\hline & 1 Space After Commas & 2 Spaces After Commas & $\beta$ & $S E$ & $t$ or $z$ statistic & $p$-value \\
\hline \multicolumn{7}{|l|}{ Early Measures } \\
\hline Skipping Rate $(\%)$ & $22.8(42.0)$ & $25.2(43.4)$ & .084 & .066 & 1.27 & .205 \\
\hline First Fixation (ms) & $220(80)$ & 217 (79) & -.017 & .010 & -1.77 & .078 \\
\hline First Pass (ms) & $301(181)$ & $297(183)$ & -.029 & .015 & -1.93 & .053 \\
\hline Number of First Pass Fixations & $1.05(0.79)$ & $1.00(0.79)$ & -.034 & .026 & -1.30 & .207 \\
\hline \multicolumn{7}{|l|}{ Late Measures } \\
\hline Regressions In (\%) & $16.8(37.4)$ & $16.9(37.5)$ & -.014 & .085 & -0.17 & .867 \\
\hline Total Time (ms) & $384(260)$ & $373(252)$ & -.022 & .016 & -1.39 & .165 \\
\hline
\end{tabular}


increasing inter-letter and inter-word spacing for readers with dyslexia, and so this group might also benefit from increased spacing near punctuation.

In sum, the current findings provide empirical evidence that supports the change made to the APA Manual specifying that two spaces should be used after the period at the end of a sentence. The initial processing of text was facilitated following two spaces and not even those who type according to the one space convention benefitted from having only one space. However, it should be noted that even though the reported effects are statistically reliable and are detectable locally at the punctuation region, they are overall small in magnitude. Punctuation spacing had no effect on the likelihood of regressing back to the punctuation region after leaving it, did not affect comprehension, and only increased overall reading speed for participants who already type according to this twospace convention (who only showed a $3 \%$ increase in overall reading speed). Thus, while period spacing does influence our processing of text, we should probably be arguing passionately about things that are more important.

Acknowledgements The order of authorship for the second and third authors was determined by a coin-toss. We would like to thank Tom Allen, Angela Persico, and Alexa Banculli for their assistance in data collection and analysis and Denis Drieghe for his helpful comments on a previous version of this manuscript. This research was funded in part with a Summer Collaborative Research Grant at Skidmore College. This work was previously presented at the 83rd Annual Meeting of the Eastern Psychological Association in Pittsburgh, PA. Correspondence concerning the paper should be addressed to Dr. Rebecca Johnson, Department of Psychology, Skidmore College, Saratoga Springs, NY 12866, USA. (email: rjohnso1@skidmore.edu).

\section{References}

American Psychological Association. (2001, Fifth Edition). Publication manual of the American Psychological Association. Washington, D.C.: American Psychological Association.

American Psychological Association. (2010, Sixth Edition). Publication manual of the American Psychological Association. Washington, D.C.: American Psychological Association.

Cui, L., Drieghe, D., Bai, X., Yan, G., \& Liversedge, S. P. (2014). Parafoveal preview benefit in unspaced and spaced Chinese reading. The Quarterly Journal Of Experimental Psychology, 67(11), 21722188. https://doi.org/10.1080/17470218.2014.909858

Drieghe, D., Brysbaert, M., \& Desmet, T. (2005). Parafoveal-on-foveal effects on eye movements in text reading: Does an extra space make a difference? Vision Research, 45, 1693-1706. https://doi.org/10. 1016/j.visres.2005.01.010

Drieghe, D., Fitzsimmons, G., \& Liversedge, S. (2017). Parafoveal preview effects in reading unspaced text. Journal of Experimental Psychology: Human Perception and Performance, 43(10), 17011716.

Epelboim, J., Booth, J. R., Ashkenazy, R., Taleghani, A., \& Steinman, R. M. (1997). Fillers and spaces in text: Evidence for the importance of word recognition during reading. Vision Research, 37, 2899-2914. https://doi.org/10.1016/S0042-6989(97)00095-3
Gonzalez, J. (August, 2014). Nothing says over 40 like two spaces after a period! [Web log message]. Retrieved from https://www. cultofpedagogy.com/two-spaces-after-period/

Grainger, J., Tydgat, I., \& Issele, J. (2010). Crowding affects letters and symbols differently. Journal of Experimental Psychology: Human Perception and Performance, 36, 673-688. https://doi.org/10.1037/ a0016888

Hill, R. L., \& Murray, W. S. (2000). Commas and spaces: Effects of punctuation on eye movements and sentence parsing. In A. Kennedy, R. Radach, D. Heller, \& J. Pynte (Eds.), Reading as a perceptual process (pp. 565-589). Oxford: Elsevier.

Hirotani, M., Frazier, L., \& Rayner, K. (2006). Punctuation and intonation effects on clause and sentence wrap-up: Evidence from eye movements. Journal of Memory and Language, 54, 425-443. https://doi. org/10.1016/j.jml.2005.12.001

Jacobs, A. (1987). On the role of blank spaces for eye-movement control in visual search. Perception \& Psychophysics, 41(5), 473-479.

Johnson, R. L., \& Eisler, M. E. (2012). The importance of the first and last letter in words during sentence reading. Acta Psychologica, 141, 336-351.

Kuznetsova, A., Brockhoff, P. B., \& Bojesen Christensen, R. H. (2015) LmerTest: Tests in Linear Mixed Effects Models.

Lee, T. (2011, January). Re: Everyone has a right to their beliefs [Web log message]. Retrieved from http://www.manifestdensity.net/2011/01/ 14/everyone-has-a-right-to-their-beliefs/

Legge, G. E., Mansfield, J. S., \& Chung, S. T. L. (2001). Psychophysics of reading XX. Linking letter recognition to reading speed in central and peripheral vision. Vision Research, 41, 725-743. https://doi.org/ 10.1016/S0042-6989(00)00295-9

Levi, D. M. (2008). Crowding: An essential bottleneck for object recognition: A mini-review. Vision Research, 48, 635-654. https://doi.org/ 10.1016/j.visres.2007.12.009

Malt, B. C., \& Seamon, J. G. (1978). Peripheral and cognitive components of eye guidance in filled-space reading. Perception \& Psychophysics, 23, 399-402.

Manjoo, F. (2011, January). Space invaders: Why you should never, ever use two spaces after a period. Slate Magazine. Retrieved from http:// www.slate.com/id/2281146/

McArdle, M. (2011, January). You can have my double space when you pry it from my cold, dead hands. The Atlantic Magazine. Retrieved from http://www.theatlantic.com/entertainment/archive/2011/01/ you-can-have-my-double-space-when-you-pry-it-from-my-colddead-hands/69592/

McConkie, G. W., \& Rayner, K. (1975). The span of the effective stimulus during a fixation in reading. Perception \& Psychophysics, 17(6), 578-586.

McGowan, V. A., White, S. J., Jordan, T. R., \& Paterson, K. B. (2014). Aging and the use of interword spaces during reading: Evidence from eye movements. Psychonomic Bulletin \& Review, 21(3), 740747. https://doi.org/10.3758/s13423-013-0527-8

McGowan, V. A., White, S. J., \& Paterson, K. B. (2015). The effects of interword spacing on the eye movements of young and older readers. Journal Of Cognitive Psychology, 27(5), 609-621. https:// doi.org/10.1080/20445911.2014.988157

Morris, R. K., Rayner, K., \& Pollatsek, A. (1990). Eye movement guidance in reading: The role of parafoveal letter and space information. Journal of Experimental Psychology: Human Perception and Performance, 16, 268-281. https://doi.org/10.1037/0096-1523.16. 2.268

Paterson, K. B., \& Jordan, T. R. (2010). Effects of increased letter spacing on word identification and eye guidance during reading. Memory \& Cognition, 38(4), 502-512. https://doi.org/10.3758/MC.38.4.502

Payne, B. R., \& Stine-Morrow, E. A. L. (2012). Aging, parafoveal preview, and semantic integration in sentence processing: Testing the cognitive workload of wrap-up. Psychology and Aging, 27, 638649. https://doi.org/10.1037/a0026540 
Pelli, D., Tillman, K. A., Freeman, J., Su, M., Berger, T. D., \& Majaj, N. J. (2007). Crowding and eccentricity determine reading rate. Journal of Vision, 7, 1-36. https://doi.org/10.1167/7.2.20

Perea, M., \& Acha, J. (2009). Space information is important for reading. Vision Research, 49, 1994-2000. https://doi.org/10.1016/j.visres. 2009.05.009

Perea, M., Moret-Tatay, C., \& Gómez, P. (2011). The effects of interletter spacing in visual-word recognition. Acta Psychologica, 137(3), 345351. https://doi.org/10.1016/j.actpsy.2011.04.003

Pollatsek, A., \& Rayner, K. (1982). Eye movement control in reading: The role of word boundaries. Journal of Experimental Psychology: Human Perception and Performance, 8(6), 817-833.

Pynte, J., \& Kennedy, A. (2007). The influence of punctuation and word class on distributed processing in normal reading. Vision Research, 47, 1215-1227. https://doi.org/10.1016/j.visres.2006.12.00

Rayner, K. (1998). Eye movements in reading and information processing: 20 years of research. Psychological Bulletin, 124, 372-422. https://doi.org/10.1037/0033-2909.124.3.372

Rayner, K. (2009). The Thirty Fifth Sir Frederick Bartlett lecture: Eye movements and attention during reading, scene perception, and visual search. Quarterly Journal of Experimental Psychology, 62, 1457-1506.

Rayner, K., Fischer, M. H., \& Pollatsek, A. (1998). Unspaced text interferes with both word identification and eye movement control. Vision Research, 38(8), 1129-1144.

Rayner, K., Kambe, G., \& Duffy, S. A. (2000). Clause wrap-up effects on eye movements during reading. Quarterly Journal of Experimental Psychology, 53, 1061-1080.

Rayner, K., McConkie, G. W., \& Zola, D. (1980). Integrating information across eye movements. Cognitive Psychology, 12(2), 206-226. https://doi.org/10.1016/0010-0285(80)90009-2

Rayner, K., Yang, J., Schuett, S., \& Slattery, T. J. (2013). Eye movements of older and younger readers when reading unspaced text. Experimental Psychology, 60, 354-361. https://doi.org/10.1027/ 1618-3169/a000207

Schotter, E. R., Angele, B., \& Rayner, K. (2012). Parafoveal processing in reading. Attention, Perception, and Psychophysics, 74, 5-35. https://doi.org/10.3758/s13414-011-0219-2
Slattery, T. J., Yates, M., \& Angele, B. (2016). Interword and interletter spacing effects during reading revisited: Interactions with word and font characteristics. Journal of Experimental Psychology: Applied, 22, 406-422. https://doi.org/10.1037/xap0000104

Spragins, A. B., Lefton, L. A., \& Fischer, D. F. (1976). Eye movements while reading and searching spatially transformed text: A developmental examination. Memory \& Cognition, 4(1), 36-42.

van den Boer, M., \& Hakvoort, B. E. (2015). Default spacing is the optimal spacing for word reading. The Quarterly Journal Of Experimental Psychology, 68(4), 697-709. https://doi.org/10.1080/ 17470218.2014.964272

Van Overshelde, J. P., \& Healy, A. F. (2005). A blank look in reading: The effect of blank space on the identification of letters and words during reading. Experimental Psychology, 52(3), 213-223.

Walker, A. (2011, January). GOOD Design Daily: Do you double space after periods? The Daily GOOD Magazine. Retrieved from http:// www.good.is/post/good-design-daily-do-you-double-space-afterperiods/

Warren, T., White, S., \& Reichle, E. (2009). Investigating the causes of wrap-up effects: Evidence from eye movements and E-Z Reader. Cognition, 111, 132-137. https://doi.org/10.1016/j.cognition.2008. 12.011

White, S. J., Warren, T. \& Reichle, E. D. (2011). Parafoveal preview during reading: Effects of sentence position. Journal of Experimental Psychology: Human Perception and Performance, 37, 1221-1238. https://doi.org/10.1037/a0022190

Wiederkehr, S. (2009, July 30). On two spaces following a period [Web log message]. Retrieved from http://blog.apastyle.org/apastyle/ 2009/07/on-two-spaces-following-a-period.html

Wolford, G., \& Chambers, L. (1983). Lateral masking as a function of spacing. Perception \& Psychophysics, 33, 129-138.

Yu, D., Cheung, S. H., Legge, G. E., \& Chung, S. T. L. (2007). Effect of letter spacing on visual span and reading speed. Journal of Vision, 7(2), 1-10. https://doi.org/10.1167/7.2.2

Zorzi, M., Barbiero, C., Facoetti, A., Lonciari, I., Carrozzi, M., Montico, M.,... Ziegler, J. C. (2012). Extra-large spacing improves reading in dyslexia. Proceedings of the National Academy of Sciences of the United States of America, 109, 11455-11459. https://doi.org/10. 1073/pnas.1205566109 